\title{
ON COMPOSITE LOOP FUNCTORS ${ }^{1}$
}

\author{
K. A. HARDIE
}

\begin{abstract}
P$ is a space with two points in a certain convenient category $\boldsymbol{C G}$ of pointed topological spaces. If $T: C G \rightarrow C G$ is a $P$ functor and $X \in C G$, we establish a homotopy equivalence $\Omega T X \simeq \Omega T * \times \Omega(X \wedge F)$, where $F$ is the fibre of $T(*): T P \rightarrow T *$.
\end{abstract}

Let $\boldsymbol{C} \boldsymbol{G}$ denote the category of compactly generated Hausdorff topological spaces with base points (denoted by $*$ ) such that, for each $X \in \boldsymbol{C} G$, $(X, *)$ has the homotopy extension property. Let $\Omega$ denote the loop space functor for pointed topological spaces. In a recent paper Gray [2] has proved the homotopy equivalence

$$
\Omega(X \vee Y) \simeq \Omega Y \times \Omega(X \times \Omega Y / \Omega Y) .
$$

The purpose of this note is to obtain a similar decomposition for $\Omega T X$, where $T: C G \rightarrow C G$ is a $P$-functor [3], [4].

Let $(W, V)$ be an NDR pair in the sense of [7] and suppose that there exists a retraction $\phi: W \rightarrow V$. For each $X \in C G$, let $T X=T_{\phi} X$ be the space obtained from $X \times W$ (i.e. the $C \boldsymbol{G}$ product) by performing the identification

$$
(*, w)=(x, \phi w) \quad(w \in W, x \in X) .
$$

Given $f: X \rightarrow Y$, let $T f\{(x, w)\}=\{(f x, w)\}$. Then we have the following

THEOREM. $T=T_{\phi}: C \boldsymbol{G} \rightarrow \boldsymbol{C G}$ is a functor and $\Omega T X \simeq \Omega T * \times \Omega(X \wedge F)$, for each $X \in \boldsymbol{C G}$, where $F$ is the fibre of $\phi$.

Let $P \in C G$ be a space with two points. There is a retraction $k$ of $P$ onto its base point. Hence if $S: C \boldsymbol{G} \rightarrow \boldsymbol{C G}$ is a functor, $S k$ is a retraction of $S P$ onto a subspace isomorphic with $S * . S$ is a $P$-functor if $S$ is naturally equivalent to $T_{\phi}$, for $\phi=S k$. (The sense differs slightly from that of [3], [4].) For example if $T X=X \vee Y, T f=f \vee i_{Y}$ (for a fixed $Y \in C G, f: X \rightarrow X^{\prime}$ ) then $T$ is a $P$-functor and applying the theorem we recover (1), for $F=$ $(\Omega Y)^{+}=P \times \Omega Y / \Omega Y$, as is observed in the proof of [2, Lemma 3]. Let $\Sigma$

Received by the editors February 25, 1972.

AMS (MOS) subject classifications (1970). Primary 55D35, 55D10, 55D05, 55E20.

Key words and phrases. Loop space, homotopy equivalence, cofibration, wedge.

${ }^{1}$ Prepared with the assistance of South African Council for Scientific and Industrial Research grant 40-332.

(c) American Mathematical Society 1973 
denote the suspension functor. Then as another application we shall obtain the following result.

COROllary. $\quad \Omega(X \times Y \mid Y) \simeq \Omega X \times \Omega(Y \wedge \Sigma \Omega X)$.

Combining the Corollary with (1) yields

$$
\Omega(X \vee Y) \simeq \Omega Y \times \Omega X \times \Omega((\Omega Y) \wedge \Sigma \Omega X),
$$

which is consistent with and may be regarded as a nonweak form of $[\mathbf{5}$, 3.7 , p. 281]:

$$
\Omega(X \vee Y) \simeq \text { (weak) } \Omega X \times \Omega Y \times \Omega(X b Y)
$$

Before proceeding to the proofs of the Theorem and Corollary we remark that the form of the Serre-Cartan construction appropriate to $\boldsymbol{C} \boldsymbol{G}$ uses the space $E X$ of Moore paths in $X$ [1] with compactly generated topology. (In this connection I wish to acknowledge a helpful conversation with Professor Eldon Dyer.) We recall that a Moore path is a pair $(f, r)$, where $r$ is a nonnegative real number and $f$ a map of the closed interval $[0, r]$ into $X$. There is a map $\lambda: X \rightarrow E X$ given by $\lambda x=(x, 0)$ which is pair-homotopy equivalent to the identity $X \rightarrow X$. It follows that $\lambda$ has the weak homotopy extension property. Moreover if we set $\mu(f, r)=$ $\min (r, 1)$ we obtain a map $\mu: E X \rightarrow I$ with the property that $\mu^{-1}(0)=\lambda(X)$. Hence, by [6, Satz I], $\lambda$ is a cofibration. If $X \in C G$, it follows that $E X \in$ $\boldsymbol{C G}$. Similarly let $\Omega^{\prime} X$ be the space of Moore loops on $X$. Since $\Omega^{\prime} X$ has the weak homotopy extension property $[9$, Satz, p. 180], a second application of [6, Satz I] shows that $\Omega^{\prime} X \in \boldsymbol{C G}$. If $F$ is the (Moore-path) fibre of $f: X \rightarrow Y$ we have a diagram

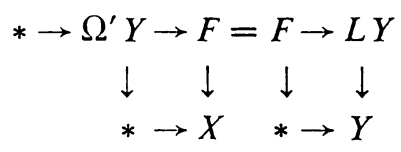

of pullback rectangles and hence by $[8$, Theorem 12] the morphisms on the top row are cofibrations. Thus $F \in \boldsymbol{C} \boldsymbol{G}$ and $L Y \in \boldsymbol{C} \boldsymbol{G}$.

Proof of THEOREM. Let $\psi=\psi X$ denote the identification map associated with (2). Then we have a diagram

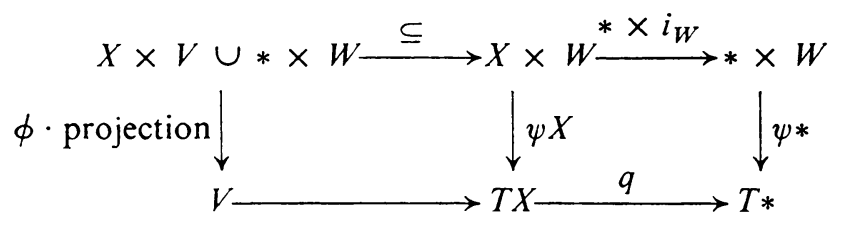

in which the composite of the bottom row is an equivalence and the lefthand rectangle is a pushout in the category of pointed topological spaces. 
Moreover an application of [7, Lemma 8.5] shows that $(T X, V)$ is an NDR pair. Since $(V, *)$ is NDR, [7, Lemma 7.2] implies that $(T X, *)$ is NDR and hence $T X \in C G$. Pulling back the Moore path fibration $L T * \rightarrow T *$ (with contractible total space) over the diagram, we obtain an upper diagram

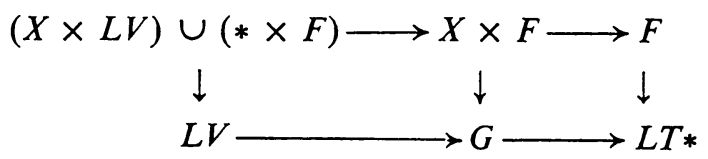

in which $G$ is the fibre of $q$ and the left-hand rectangle is again a pushout. (If in $\boldsymbol{C} \boldsymbol{G}$ a map is pulled back over the pushout of a cofibration then the upper diagram is necessarily a pushout.) Since $(G, L V)$ and $(X \times F$, $X \times L V \cup * \times F$ ) are NDR by [8, Theorem 12], we have

$$
\begin{aligned}
G \simeq G / L V & \approx X \times F /(X \times L V \cup * \times F) \\
& \simeq X \times F /(X \times * \cup * \times F)=X \wedge F .
\end{aligned}
$$

But $q$ is a retraction and hence its Serre-Cartan fibration has a cross section. As in [2, Lemma 2] we may obtain $\Omega^{\prime} T X \simeq \Omega^{\prime} G \times \Omega^{\prime} T *$, completing the proof.

Proof of Corollary. For a fixed $X \in C G$, let $T Y=X \times Y / Y$. Certainly $T$ is a $P$-functor. Moreover $\phi$ is the folding map $X \vee X \rightarrow X$ and it is easily shown that $F \simeq \Sigma \Omega X$.

\section{REFERENCES}

1. J. F. Adams and P. J. Hilton, On the chain algebra of a loop space, Comment. Math. Helv. 30 (1956), 305-330. MR 17, 1119.

2. B. Gray, A note on the Hilton-Milnor theorem, Topology 10 (1971), 199-201. MR 43 \#6921.

3. K. A. Hardie, Weak homotopy equivalence of P-functors, Quart. J. Math. Oxford Ser. (2) 19 (1968), 17-31. MR 37 \#3564.

4. - Homotopy of natural transformations, Canad. J. Math. 22 (1970), 332-341. MR 41 \#6209.

5. P. J. Hilton, Homotopy theory of modules and duality, Symposium Internacional de Topologia Algebraica, Universidad Nacional Autonoma de Mexico and UNESCO, Mexico City, 1958, pp. 273-281. MR 20 \#4588.

6. D. Puppe, Bemerkungen über die Erweiterung von Homotopien, Arch. Math. (Basel) 18 (1967), 81-88. MR 34 \#6770.

7. N. E. Steenrod, A convenient category of topological spaces, Michigan Math. J. 14 (1967), 133-152. MR 35 \#970.

8. A. Strøm, Note on cofibrations. II, Math. Scand. 22 (1968), 130-142. MR 39 \#4846.

9. T. tom Dieck, K. H. Kamps and D. Puppe, Homotopietheorie, Lecture Notes in Math., vol. 157, Springer-Verlag, Berlin and New York, 1970.

Department of Mathematics, University of Cape Town, Rondebosch, C.P., SOUTH AFRICA 